\title{
Hanbury Brown-Twiss Effect with Wave Packets
}

\author{
Tabish Qureshi ${ }^{1}$ \& Ushba Rizwan ${ }^{2}$ \\ ${ }^{1}$ Centre for Theoretical Physics, Jamia Millia Islamia, New Delhi, India. E-mail:|tabish@ctp-jamia.res.in \\ ${ }^{2}$ Department of Physics, Jamia Millia Islamia, New Delhi, India. E-mail:93.ushba@gmail.com
}

Editors: Radu lonicioiu \& Danko Georgiev

Article history: Submitted on October 27, 2017; Accepted on November 28, 2017; Published on November 30, 2017.

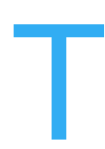
he Hanbury Brown-Twiss (HBT) effect, at the quantum level, is essentially an interference of one particle with another, as opposed to interference of a particle with itself. Conventional treatments of identical particles encounter difficulties while dealing with entanglement. A recently introduced label-free approach to indistinguishable particles is described, and is used to analyze the HBT effect. Quantum wave-packets have been used to provide a better understanding of the quantum interpretation of the HBT effect. The effect is demonstrated for two independent particles governed by Bose-Einstein or Fermi-Dirac statistics. The HBT effect is also analyzed for pairs of entangled particles. Surprisingly, entanglement has almost no effect on the interference seen in the HBT effect. In the light of the results, an old quantum optics experiment is reanalyzed, and it is argued that the interference seen in that experiment is not a consequence of non-local correlations between the photons, as is commonly believed.

Quanta 2017; 6: 61-69.

\section{Introduction}

Interference of waves is a very old and well studied subject. Waves emanating from two sources give rise to interference, provided that there is a coherence in the phases of the two. This can be understood with the following argument. Suppose there are two sources A and B, waves emerging from which fall on a distant screen. At a point $x_{1}$ on the screen, the contribution of the two classical waves can be written as

$$
\mathcal{E}\left(x_{1}\right)=\alpha e^{\imath k r_{A 1}}+\beta e^{\imath k r_{B 1}-l \phi},
$$

where $k$ is the wave-vector of the two waves, $r_{A 1}, r_{B 1}$ are the displacements as shown in Figure 1, and $\phi$ is the difference in the phases of the two sources. Assuming $\alpha, \beta$ to be real, the intensity at the point $x_{1}$ is then given by

$I\left(x_{1}\right) \equiv\left|\mathcal{E}\left(x_{1}\right)\right|^{2}=\alpha^{2}+\beta^{2}+2 \alpha \beta \cos \left[k\left(r_{A 1}-r_{B 1}\right)+\phi\right]$.

The above expression represents interference between the wave from $\mathrm{A}$ and $\mathrm{B}$, at point $x_{1}$ on the screen. One can see that if the phase difference between the two waves, $\phi$, fluctuates randomly with time, which amounts to integrating the above over $\phi$, the interference will be washed away.

$$
\left\langle I\left(x_{1}\right)\right\rangle \approx \alpha^{2}+\beta^{2} .
$$

Thus two independent, incoherent sources of waves do not give rise to interference. Hanbury Brown and Twiss carried out an experiment with radio waves which involved correlating intensities at two points on the screen, coming from two independent sources [1]. Their experiment can 


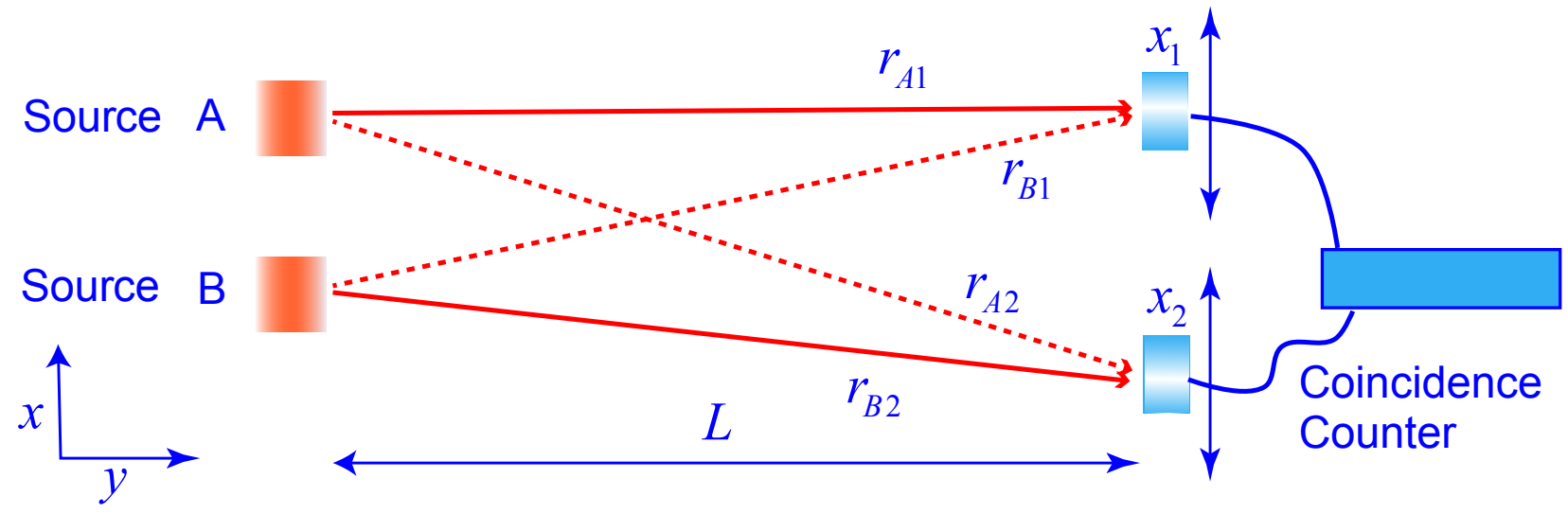

Figure 1: A schematic diagram for a two source interference, and also for a intensity correlation at two points. Sources A and B are stationary while the detectors at $x_{1}$ and $x_{2}$ are movable.

be easily understood from the preceding example. An intensity correlation at two points $x_{1}$ and $x_{2}$ on the screen can be written as

$$
\begin{aligned}
I\left(x_{1}\right) I\left(x_{2}\right)= & \left|\mathcal{E}\left(x_{1}\right)\right|^{2}\left|\mathcal{E}\left(x_{2}\right)\right|^{2} \\
= & {\left[\alpha^{2}+\beta^{2}+2 \alpha \beta \cos \left(k\left(r_{A 1}-r_{B 1}\right)+\phi\right)\right] } \\
& {\left[\alpha^{2}+\beta^{2}+2 \alpha \beta \cos \left(k\left(r_{A 2}-r_{B 2}\right)+\phi\right)\right] }
\end{aligned}
$$

After averaging over $\phi$, the $\phi$-dependent terms drop out, and one is left with

$$
\begin{aligned}
\left\langle I\left(x_{1}\right) I\left(x_{2}\right)\right\rangle \approx & \left(\alpha^{2}+\beta^{2}\right)^{2} \\
& +2(\alpha \beta)^{2} \cos \left(k\left(r_{A 1}-r_{B 1}-r_{A 2}+r_{B 2}\right)\right) .
\end{aligned}
$$

Surprisingly, although individual intensities do not show any oscillation, there is interference between the intensities at two different points on the screen. This is what is called the Hanbury Brown-Twiss (HBT) effect. Random fluctuation of phases of the independent source has no effect on the oscillations seen in the intensity correlations. Notice that the visibility [2] of the above interference pattern is $\frac{2(\alpha \beta)^{2}}{\left(\alpha^{2}+\beta^{2}\right)^{2}}$ which is bounded from above by $1 / 2$.

The HBT effect was explained using classical waves, but when the same experiment was proposed using light, there was a lot of skepticism, and people thought it would not work. The reason was that the HBT effect arose from two different waves interfering with each other, and many people believed that a photon interferes only with itself, two photons never interfere with each other [3, p. 9]. Hanbury Brown and Twiss carried out the experiment with light and demonstrated the HBT effect [4]. The first quantum understanding of the HBT effect for light was given by Fano [5]. The HBT effect has now been demonstrated for quantum light [6,7]. Not only that, it has also been demonstrated with massive particles, both of bosonic [8-10] and fermionic [11] nature.
The HBT effect for massive particles is intriguing, as it involves interference between two different particles. Particles are seen to be bunched together without any interaction between them. It is a purely quantum mechanical effect, and the classical wave explanation is only a part of the story. Full treatment of the HBT effect can of course be done using quantum many-body theory, as the particles are treated as being indistinguishable there. However, that maybe an overkill, as it is essentially a two particle effect. In the following we treat two massive particles as traveling wave-packets, and use them to analyze the HBT effect.

\section{Label-free approach to identical particles}

For a quantum treatment of the HBT effect, it is essential to take the indistinguishability of the particles into account. However, treatment of identical particles in quantum mechanics, particularly those involving entanglement, has been an issue which has been much debated [12-29]. The problem can be seen in the very basic symmetric or anti-symmetric wavefunctions that are usually written, e.g. $\phi_{A}\left(x_{1}\right) \phi_{B}\left(x_{2}\right) \pm \phi_{B}\left(x_{1}\right) \phi_{A}\left(x_{2}\right)$. Although the particles are assumed to be indistinguishable, we put labels 1 and 2 on them. In addition, since the above state is not separable, the question arises, whether it is an entangled state? The problem of labeling, of course, disappears if one uses the second quantization approach [30]. However, the problems in dealing with entangled states do not go away just by using second quantization. Recently a new state-based approach has been introduced which does not label the particles, and appears to address the entanglement related issues satisfactorily [31]. In the following, we will explain this approach and use it to analyze HBT experiment with quantum particles. 
In this new approach, the basic assumption is that in dealing with more than one identical particles, the particles cannot be individually addressed. This is in accordance with the quantum theory. The combined state of two particles, which may consist of single-particle states, is treated as a holistic indivisible entity [31]. One cannot ask for the form of this state. What one can ask, is what the two particle probabiliity amplitude of finding the two particles in two different states is. For example, if one particle is in the single-particle state $|\psi\rangle$ and one in the state $|\phi\rangle$, the two-particle state is represented as $|\phi, \psi\rangle$. One may not talk about the "form" of this state in terms of single-particle states $|\psi\rangle$ and $|\phi\rangle$. However, the probability amplitude of finding one particle in state $|\alpha\rangle$ and the other in state $|\beta\rangle$, i.e., in the combined state $|\alpha, \beta\rangle$, is given by

$$
\langle\alpha, \beta \mid \phi, \psi\rangle=\langle\alpha \mid \phi\rangle\langle\beta \mid \psi\rangle+\eta\langle\alpha \mid \psi\rangle\langle\beta \mid \phi\rangle,
$$

where $\eta^{2}=1$. One can check that the state $|\phi, \psi\rangle$ is not normalized simply by replacing $|\alpha, \beta\rangle$ by $|\phi, \psi\rangle$ in the above equation. The state $|\phi, \psi\rangle$ has to be multiplied with $\frac{1}{\sqrt{1+\eta|\langle\phi \mid \psi\rangle|^{2}}}$ in order to normalize it. The probability amplitude of finding the particles at positions $x_{1}$ and $x_{2}$, i.e., in the state $\left|x_{1}, x_{2}\right\rangle$ is given by

$$
\left\langle x_{1}, x_{2} \mid \phi, \psi\right\rangle=\left\langle x_{1} \mid \phi\right\rangle\left\langle x_{2} \mid \psi\right\rangle+\eta\left\langle x_{1} \mid \psi\right\rangle\left\langle x_{2} \mid \phi\right\rangle,
$$

which is the familiar symmetric or antisymmetric twoparticle wavefunction. The difference is that, in this case 1 and 2 are not labels of particles, but corresponds to the two positions of a joint measurement.

A one-particle operator $\hat{A}$ acts on the two-particle state in the following way:

$$
\hat{A}|\phi, \psi\rangle=|\hat{A} \phi, \psi\rangle+|\phi, \hat{A} \psi\rangle .
$$

The expectation value of a one-particle operator, using (6) and (8), is given by

$$
\begin{aligned}
\langle\hat{A}\rangle= & \langle\phi, \psi|\hat{A}| \phi, \psi\rangle \\
= & \langle\phi \mid \hat{A} \phi\rangle+\langle\psi \mid \hat{A} \psi\rangle \\
& +\eta(\langle\phi \mid \psi\rangle\langle\psi \mid \hat{A} \phi\rangle+\langle\psi \mid \phi\rangle\langle\phi \mid \hat{A} \psi\rangle),
\end{aligned}
$$

which agrees with the expression of the conventional analysis.

\section{Independent particles}

Let there be two particles described by two wave packets, traveling along y-axis. The two particles emerge from two sources localized at positions $x_{0}$ and $-x_{0}$. We described the two particles by two Gaussian wave packets of width $\epsilon$ each, localized at $x_{0}$ and $-x_{0}$, denoted by $\left|\phi_{A}\right\rangle$ and $\left|\phi_{B}\right\rangle$, respectively. Since the particles are indistinguishable, the combined initial state of the two particles can be written as

$$
|\psi(0)\rangle=\left|\phi_{A}, \phi_{B}\right\rangle
$$

This satisfies the essential requirement for HBT effect, that the particles be identical, in the quantum sense. The conventional two-particle wavefunction is then just the probability amplitude of finding one particle at $x_{1}$ and one at $x_{2}$, and can be written down using (6) as follows:

$$
\begin{aligned}
\psi\left(x_{1}, x_{2}, 0\right)= & \left\langle x_{1}, x_{2} \mid \phi_{A}, \phi_{B}\right\rangle \\
= & \left\langle x_{1} \mid \phi_{A}\right\rangle\left\langle x_{2} \mid \phi_{B}\right\rangle+\eta\left\langle x_{1} \mid \phi_{B}\right\rangle\left\langle x_{2} \mid \phi_{A}\right\rangle \\
= & \frac{1}{\sqrt{\pi} \epsilon}\left(e^{\frac{-\left(x_{1}-x_{0}\right)^{2}}{\epsilon^{2}}} e^{\frac{-\left(x_{2}+x_{0}\right)^{2}}{\epsilon^{2}}}\right. \\
& \left.+\eta e^{\frac{-\left(x_{1}+x_{0}\right)^{2}}{\epsilon^{2}}} e^{\frac{-\left(x_{2}-x_{0}\right)^{2}}{\epsilon^{2}}}\right)
\end{aligned}
$$

where $\eta= \pm 1$. The last line in the above equation specifies the Gaussian form of the states emerging from the sources $\mathrm{A}$ and $\mathrm{B}$, namely, $\phi_{A}(x)=\exp \frac{-\left(x-x_{0}\right)^{2}}{\epsilon^{2}}$ and $\phi_{B}(x)=\exp \frac{-\left(x+x_{0}\right)^{2}}{\epsilon^{2}}$. For bosonic particles, the wavefunction should be symmetric, and $\eta$ should be 1 . For fermions, the two-particle wavefunction should be antisymmetric, requiring $\eta$ to be -1 . We assume that the particles are traveling along the positive $y$-direction with a constant velocity $v_{0}$. For simplicity we ignore the explicit time evolution along the $y$-axis, and assume that evolution for a time $t^{\prime}$ just transports the wave packets by a distance $l=v_{0} t^{\prime}$. The dispersion of the wave packets along the transverse $x$-direction is more interesting and may give rise to interference between wave packets.

Notice that if one of the two sources produces a wave packet with an additional phase factor, say $e^{l \phi}$, that phase factor will present in the both the terms in (11), and can be pulled out, becoming irrelevant.

The Hamiltonian governing the time evolution is that of two free particles. The two-particle eigenstate will simply be $\left|p, p^{\prime}\right\rangle$ which means one particle has momentum $p$ and the other $p^{\prime}$. One can thus write

$$
\hat{H}\left|p, p^{\prime}\right\rangle=\left(\frac{p^{2}}{2 m}+\frac{p^{\prime 2}}{2 m}\right)\left|p, p^{\prime}\right\rangle
$$

After traveling for a time $t$, the particles reach the screen. Time evolution of the initial state $\left|\phi_{A}, \phi_{B}\right\rangle$ can be worked out by introducing a complete set of two-particle momen- 
tum eigenstates:

$$
\begin{aligned}
|\psi(t)\rangle= & e^{-l \hat{H} t / \hbar}\left|\phi_{A}, \phi_{B}\right\rangle \\
= & e^{-l \hat{H} t / \hbar} \sum_{p, p^{\prime}}\left|p, p^{\prime}\right\rangle\left\langle p, p^{\prime} \| \phi_{A}, \phi_{B}\right\rangle \\
= & \sum_{p, p^{\prime}} e^{-l \frac{\left(p^{2}+p^{\prime 2}\right) t}{2 m \hbar}}\left|p, p^{\prime}\right\rangle \\
= & \left.\sum_{p, p^{\prime}}\left|p, \phi_{A}\right\rangle\left\langle p^{\prime} \mid \phi_{B}\right\rangle+\eta\left\langle p \mid \phi_{B}\right\rangle\left\langle p^{\prime} \mid \phi_{A}\right\rangle\right] \\
& {\left[\left\langle p \mid \phi_{A}(t)\right\rangle\left\langle p^{\prime} \mid \phi_{B}(t)\right\rangle+\eta\left\langle p \mid \phi_{B}(t)\right\rangle\left\langle p^{\prime} \mid \phi_{A}(t)\right\rangle\right] } \\
= & \left|\phi_{A}(t), \phi_{B}(t)\right\rangle,
\end{aligned}
$$

where

$$
\left\langle p \mid \phi_{A}(t)\right\rangle=e^{-\frac{i p^{2} t}{2 m \hbar}}\left\langle p \mid \phi_{A}\right\rangle, \quad\left\langle p^{\prime} \mid \phi_{B}(t)\right\rangle=e^{-\frac{i p^{2} t}{2 m \hbar}}\left\langle p \mid \phi_{B}\right\rangle .
$$

From the momentum representation of $\left|\phi_{A}\right\rangle$ and $\left|\phi_{B}\right\rangle$, the position representation can be evaluated. The amplitude of finding the particles at positions $x_{1}$ and $x_{2}$ then works out to be

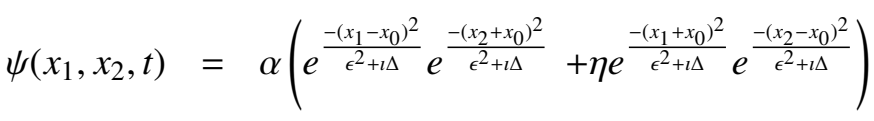

where $\Delta \equiv 2 \hbar t / m$, and $\alpha=\frac{1}{\sqrt{\pi(\epsilon+l \Delta / \epsilon)}}$. For simplicity we introduce the notation $\psi_{t}=\psi\left(x_{1}, x_{2}, t\right)$. The joint probability density of finding the particles at $x_{1}$ and $x_{2}$ is given by

$$
\begin{aligned}
\left|\psi_{t}\right|^{2}= & \frac{1}{\pi \sigma^{2}}\left(e^{\frac{-2 \epsilon^{2}\left(x_{1}-x_{0}\right)^{2}}{\epsilon^{4}+\Delta^{2}}} e^{\frac{-2 \epsilon^{2}\left(x_{2}+x_{0}\right)^{2}}{\epsilon^{4}+\Delta^{2}}}\right. \\
& +e^{\frac{-2 \epsilon^{2}\left(x_{1}+x_{0}\right)^{2}}{\epsilon^{4}+\Delta^{2}}} e^{\frac{-2 \epsilon^{2}\left(x_{2}-x_{0}\right)^{2}}{\epsilon^{4}+\Delta^{2}}} \\
& +\eta e^{\frac{-\left(x_{1}+x_{0}\right)^{2}}{\epsilon^{2}+l \Delta}} e^{\frac{-\left(x_{2}-x_{0}\right)^{2}}{\epsilon^{2}+l \Delta}} e^{\frac{-\left(x_{1}-x_{0}\right)^{2}}{\epsilon^{2}-l \Delta}} e^{\frac{-\left(x_{2}+x_{0}\right)^{2}}{\epsilon^{2}-l \Delta}} \\
& \left.+\eta e^{\frac{-\left(x_{1}-x_{0}\right)^{2}}{\epsilon^{2}+l \Delta}} e^{\frac{-\left(x_{2}+x_{0}\right)^{2}}{\epsilon^{2}+l \Delta}} e^{\frac{-\left(x_{1}+x_{0}\right)^{2}}{\epsilon^{2}-l \Delta}} e^{\frac{-\left(x_{2}-x_{0}\right)^{2}}{\epsilon^{2}-l \Delta}}\right)
\end{aligned}
$$

where $\sigma^{2}=\epsilon^{2}+\Delta^{2} / \epsilon^{2}$. The above simplifies to

$$
\begin{aligned}
\left|\psi_{t}\right|^{2}= & \frac{2}{\pi \sigma^{2}} e^{\frac{-2 \epsilon^{2}\left(x_{1}^{2}+x_{2}^{2}+2 x_{0}^{2}\right)}{\epsilon^{4}+\Delta^{2}}} \cosh \left[\frac{4 \epsilon^{2}\left(x_{1}-x_{2}\right) x_{0}}{\epsilon^{4}+\Delta^{2}}\right] \\
& \left(1+\eta \frac{\cos \left(\frac{4 \Delta\left(x_{1}-x_{2}\right) x_{0}}{\epsilon^{4}+\Delta^{2}}\right)}{\cosh \left(\frac{4 \epsilon^{2}\left(x_{1}-x_{2}\right) x_{0}}{\epsilon^{4}+\Delta^{2}}\right)}\right) .
\end{aligned}
$$

The right-hand side of Eq. (17) represents an interference pattern in the joint probability of detection of the two particles, with respect to the distance between the two positions $x_{1}$ and $x_{2}$ on the screen. For $\eta=1$, it constitutes the HBT effect for massive particles, which obey Bose statistics. The same result will also apply to independent photons with the proviso that $\Delta=\lambda L / \pi$, where $\lambda$ is the wave-length of the photons and $L$ is the distance traveled by them in time $t$. In fact, the relation $\Delta=\lambda L / \pi$ may also be used for massive particles in which case $\lambda$ will represent the de Broglie wavelength of a particle. It is easy to check that, for $\left|x_{1}-x_{2}\right|, x_{0} \ll r_{A 1}+r_{A 2}, r_{B 1}+r_{B 2}$, the cosine term in (5) reduces to $\cos \left(4 x_{0}\left(x_{1}-x_{2}\right) / \Delta\right)$, which is the same as the cosine term in (17), provided that $\epsilon^{4} \ll \Delta^{2}$.

At this stage it may be useful to understand the physical meaning of various terms in the joint probability distribution (16). In our usual classical way of thinking, we imagine that there is a possibility of the particle from source A reaching $x_{1}$ and that from source B reaching $x_{2}$. The solids lines in Figure 1 represents this possibility and the first term in (16) represents its probability. There is also the possibility of the particle from source A reaching $x_{2}$ and that from source B reaching $x_{1}$. The dashed lines in Figure 1 represent this possibility and the second term in (16) represents its probability. However, in quantum mechanics particles are not localized objects. In fact they are capable of demonstrating wave and particle natures in different situations. They should rather be called quantons [32]. Quantons may be visualized as fuzzy objects, sometime being spreadout like a wave, and sometimes localized as particles. They can very well interfere with themselves. If two independent quantons can interfere with each other, there is also the possibility of quantons from $\mathrm{A}$ and $\mathrm{B}$ partially contributing to the quanton detected at $x_{1}$, and at $x_{2}$. Since the quantons are identical, we have no way of knowing which source they came from. In Figure 1, the solid line $r_{A 1}$ and the dotted line $r_{B 1}$ represent the possibility of A and B contributing to the quantons reaching $x_{1}$, and the solid line $r_{B 2}$ and the dotted line $r_{A 2}$ represent the possibility of $\mathrm{B}$ and $\mathrm{A}$ contributing to the quantons reaching $x_{2}$. The last two terms in (16) represent this possibility. One can see that if the quantons are not identical, the last two terms would not be there. This argument is in agreement with the fact that the HBT effect cannot be seen for particles which are not identical.

The last two terms can also be interpreted as describing interference between the processes represented by the solid and the dashed lines. For certain values of $x_{1}, x_{2}$ it might so happen that the solid lines process and the dashed lines process destructively interfere. In that case, there will be no simultaneous detection of quantons at all.

The visibility of this interference pattern is $\frac{1}{\cosh \left(\frac{4 \epsilon^{2}\left(x_{1}-x_{2}\right) x_{0}}{\epsilon^{4}+\Delta^{2}}\right)}$, which is bounded from above by 1 . Contrast this with the classical HBT effect described by (5), where the visibility cannot be greater than $1 / 2$. 
This implies that there are certain distances between the detectors 1 and 2 for which the probability of detecting particles simultaneously is zero! The probability of detecting two particles very close to each other is enhanced. This can be interpreted as a bunching effect. Remarkably, the particles tend to get bunched together even when there is no interaction between them. This is a purely quantum mechanical effect and has been experimentally observed in photons [6,7] as well as massive particles [8-10].

For $\eta=-1$, the relation (17) implies that the probability of simultaneously detecting two particles very close to each other is nearly zero! Even when the particles strike the screen at random, there is a certain probability of two of them hitting the screen very close to each other. In the case $\eta=-1$, the probability of landing very close to each other is even smaller than this random chance. It appears as if the particles are repelling each other. This is what is called anti-bunching effect and has been observed for particles following Fermi-Dirac statistics, e.g., electrons [11].

\section{Entangled particles}

Let us now investigate the scenario where the particles are entangled. There are certain sources of photons which generate photons in entangled pairs. Entanglement manifests itself in strong quantum correlations between the two particles. To our knowledge, the effect of entanglement on the HBT effect has not been quantified. Einstein, Podolsky and Rosen (EPR) first drew attention to a momentum entangled state of two particles [33]

$$
\Psi_{\mathrm{EPR}}\left(x_{1}, x_{2}\right)=\int_{-\infty}^{\infty} e^{\frac{i p x_{1}}{\hbar}} e^{\frac{-i p x_{2}}{\hbar}} e^{\frac{-l 2 x_{0} p}{\hbar}} d p,
$$

which can be written in Dirac notation as

$$
\left|\Psi_{\mathrm{EPR}}\right\rangle=\int_{-\infty}^{\infty}|p\rangle_{1}|-p\rangle_{2} e^{\frac{-12 x_{0} p}{\hbar}} d p,
$$

where labels 1 and 2 refer to particle 1 and 2, respectively. This state is for distinguishable particles. If one were to write an EPR state for identical particles, in our label-free approach, it would be the following

$$
\left|\Psi_{\mathrm{EPR}}^{\text {ident }}\right\rangle=\int_{-\infty}^{\infty}|p,-p\rangle e^{\frac{-12 x_{0} p}{\hbar}} d p .
$$

The amplitude of finding the particles at $x_{1}$ and $x_{2}$ is given by

$$
\begin{aligned}
\left\langle x_{1}, x_{2} \mid \Psi_{\mathrm{EPR}}^{\text {ident }}\right\rangle= & \int_{-\infty}^{\infty}\left[\left\langle x_{1} \mid p\right\rangle\left\langle x_{2} \mid-p\right\rangle\right. \\
& \left.+\eta\left\langle x_{1} \mid-p\right\rangle\left\langle x_{2} \mid p\right\rangle\right] e^{\frac{-12 x_{0} p}{\hbar}} d p,
\end{aligned}
$$

which will be just the symmetrized or antisymmetrized form of $(18)$.

The problem with the EPR state (18) is that it cannot be normalized, and also it does not describe particles with varying degree of entanglement. To address these shortcomings, we introduce a generalized EPR state for identical particles

$|\Psi\rangle=C \int_{-\infty}^{\infty} \int_{-\infty}^{\infty}|q+p, q-p\rangle e^{\frac{-12 x_{0} p}{\hbar}} e^{-\frac{p^{2}}{\hbar^{2} \sigma^{2}}} e^{-\frac{q^{2} \Omega^{2}}{\hbar^{2}}} d p d q$,

where $q+p$ and $q-p$ label single-particle momentum eigenstates, $C$ is a normalization constant, and $\sigma, \Omega$ are certain parameters. In the limit $\sigma, \Omega \rightarrow \infty$ the state 22 reduces to the EPR state (20), if $-2 x_{0}$ here is identified with $x_{0}$ in the EPR state.

The two-particle amplitude of finding them at $x_{1}$ and $x_{2}$ is given by

$$
\begin{aligned}
\Psi\left(x_{1}, x_{2}\right)= & \sqrt{\frac{\sigma}{\pi \Omega}}\left(e^{-\left(x_{1}-x_{2}-2 x_{0}\right)^{2} \sigma^{2}} e^{-\left(x_{1}+x_{2}\right)^{2} / 4 \Omega^{2}}\right. \\
& \left.+\eta e^{-\left(x_{1}-x_{2}+2 x_{0}\right)^{2} \sigma^{2}} e^{-\left(x_{1}+x_{2}\right)^{2} / 4 \Omega^{2}}\right) .
\end{aligned}
$$

The state $(23)$ is an extended version of the generalized EPR state introduced earlier [34]. It is straightforward to show that $\Omega$ and $\sigma$ quantify the position and momentum spread of the particles in the $x$-direction. The interesting thing about this state is that for $2 \Omega=1 / \sigma=\epsilon \sqrt{2}$, it is no longer entangled and reduces exactly to the symmetric state (11) studied in the last section, which is a symmetrized or anti-symmetrized product of two Gaussians centered at $x_{0}$ and $-x_{0}$. So the entangled state is essentially two shifted Gaussians entangled with each other. The state (23) is symmetric under the interchange of the two particles, thus describing bosonic particles.

The stage is now set to study HBT effect with two entangled particles, described by the state (23). The two particles travel in the $y$-direction for a time $t$ before reaching the screen. During this time, the states evolves in transverse $x$-direction too. As done in the last section, we ignore the time evolution in the $y$-direction, and only consider the evolution in the $x$-direction. If one is dealing with photons, one can use an alternative wave-packet evolution [35]. The state of the two particles, on reaching the screen (or detectors), is given by

$$
\begin{aligned}
\Psi\left(x_{1}, x_{2}, t\right)= & C_{t} e^{\left[-\frac{\left(x_{1}+x_{2}\right)^{2}}{4 \Omega^{2}+l \delta}\right]}\left(\exp \left[-\frac{\left(x_{1}-x_{2}-2 x_{0}\right)^{2}}{1 / \sigma^{2}+l \delta}\right]\right. \\
& \left.+\eta \exp \left[-\frac{\left(x_{1}-x_{2}+2 x_{0}\right)^{2}}{1 / \sigma^{2}+\imath \delta}\right]\right)
\end{aligned}
$$

where $C_{t}=\sqrt{\frac{1}{\pi}}\left[\left\{\Omega^{2}+\left(\frac{\lambda L}{2 \pi \Omega}\right)^{2}\right\}\left\{\frac{1}{\sigma^{2}}+\left(\frac{2 \sigma \lambda L}{\pi}\right)^{2}\right\}\right]^{-1 / 4}$, $\delta=4 \hbar t / m=2 \lambda L / \pi$ and $L$ is the distance in the $y$-direction, traveled by the particles during time $t$. 


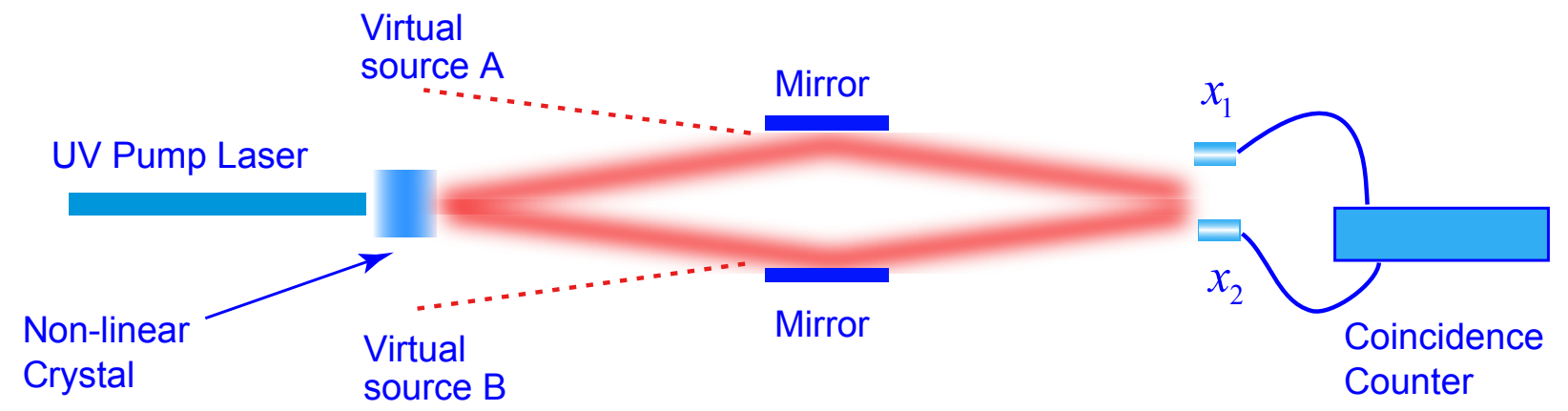

Figure 2: A schematic diagram of the Ghosh-Mandel experiment [36]. A UV pump laser falls on a non-linear crystal and produces pairs of entangled photons via spontaneous parametric down-conversion (SPDC), traveling in different directions. These photons are deflected by two mirrors and recombined where two movable detectors detect them in coincidence.

The probability density of joint detection of particles at $x_{1}$ and $x_{2}$ can now be calculated, and is given by

$$
\begin{aligned}
\left|\Psi\left(x_{1}, x_{2}, t\right)\right|^{2}= & \left|C_{t}\right|^{2} e^{\frac{-8 \Omega^{2}\left(x_{1}+x_{2}\right)^{2}}{16 \Omega^{4}+\delta^{2}}} e^{\frac{-2\left(\left(x_{1}-x_{2}\right)^{2}+2 x_{0}^{2}\right) / \sigma^{2}}{1 / \sigma^{4}+\delta^{2}}} \\
& \cosh \left[\frac{8\left(x_{1}-x_{2}\right) x_{0} / \sigma^{2}}{1 / \sigma^{4}+\delta^{2}}\right] \\
& \left(1+\eta \frac{\cos \left(\frac{8 \delta\left(x_{1}-x_{2}\right) x_{0}}{1 / \sigma^{4}+\delta^{2}}\right)}{\cosh \left[\frac{8\left(x_{1}-x_{2}\right) x_{0} / \sigma^{2}}{1 / \sigma^{4}+\delta^{2}}\right]}\right)
\end{aligned}
$$

The above expression closely resembles (17) derived for particles which are not entangled. Let us explore it in the situation when the entanglement between the two particles is strong. From the Gaussian in $\left(x_{1}-x_{2}\right)$ in (23), one can see that as $\sigma$ increases, the Gaussian narrows, and $\left(x_{1}-x_{2}-2 x_{0}\right)$ becomes more localized, which implies stronger position correlation between detected particles. Stronger position correlation implies stronger entanglement. Thus, entanglement is strong when $\sigma$ is large and one can safely assume $1 / \sigma^{4} \ll \delta^{2}$. In this limit, the cosine term becomes $\cos \left[8\left(x_{1}-x_{2}\right) x_{0} / \delta\right]=\cos \left[4\left(x_{1}-x_{2}\right) x_{0} / \Delta\right]$, which means that the interference fringe width is the same as in the case of independent particles and also in the classical case. The Gaussian terms in 25 assume the approximate form

$$
e^{\frac{-2\left(x_{1}+x_{2}\right)^{2}}{4 \Omega^{2}+\delta^{2} / 4 \Omega^{2}}} e^{\frac{-2\left(\left(x_{1}-x_{2}\right)^{2}+2 x_{0}^{2}\right)}{\sigma^{2} \delta^{2}}}
$$

These Gaussians represent broad profiles both in $x_{1}+x_{2}$ and $x_{1}-x_{2}$. Therefore it appears that entanglement does not cause any suppression of HBT effect, and remains almost as it is for independent particles.

In the opposite limit, i.e., when the entanglement goes to zero, one can write $4 \Omega^{2}=1 / \sigma^{2} \equiv 2 \epsilon^{2}$ (say). In this limit, (25) exactly reduces to (17), as expected.

\section{The Ghosh-Mandel Experiment}

In the light of the preceding analysis, we now take a fresh look at an old quantum optics experiment by Ghosh and Mandel [36]. This experiment was among the category of first experiments showing spatial correlation of photons. A UV laser beam was incident on a non-linear crystal resulting in the production of a pair of photons via spontaneous parametric down-conversion (SPDC). Such photons are known to be entangled, and show quantum correlations. The two photons travel in different directions at a small angle with respect to each other. Two mirrors are used to bring the two photons together, and two detectors, in the detection plane, detect them in coincidence (see Figure 2). While a single detector saw no interference, a coincident count of the two detectors, as a function of their relative separation showed an interference pattern.

The visibility of interference was greater than $1 / 2$, which demonstrated the non-classical nature of photons. This experiment has also been discussed in a textbook [37], and the interference pattern is believed to be a result of non-local quantum correlation between the two photons [36, 37].

One would notice the close similarity between the Ghosh-Mandel experiment and our model system studying HBT effect with entangled particles. The two detectors in the Ghosh-Mandel experiment, at $x_{1}$ and $x_{2}$, see the photons reaching them after getting deflected from the two mirrors. In effect they see the photons as coming from two spatially separated virtual sources A and B (see Figure 2). With this recognition, the setup in Figure 2 is virtually the same as that in Figure 1 , and our model system captures the essence of the Ghosh-Mandel experiment. One might wonder if the generalized EPR state can describe the state of entangled photons emerging from an SPDC. In fact, the state of the SPDC photons, produced 
from a Gaussian pump beam, is closely similar to the generalized EPR state (23) if one puts $x_{0}$ equal to zero [38]. The effect of the two mirrors in the Ghosh-Mandel experiment (see Figure 2), is to make the two photons appear to arrive from two spatially separated locations. Hence, the introduction of $x_{0}$ in $(23)$ incorporates the effect of the two mirrors into the state of the entangled photons.

Our analysis shows interference of entangled particles, and the visibility is close to 1 . So it reproduces the result of the Ghosh-Mandel experiment. However, the surprising part is that the same result is obtained when we use independent bosonic particles which are not entangled, as shown in Section 3. When independent bosonic particles are used, one sees an interference in the coincidence count as a function of the relative position of the detectors, and the visibility is close to 1 . But that is the result that is obtained in the Ghosh-Mandel experiment too. This implies that in the Ghosh-Mandel experiment, if the photons pairs were not entangled, the result would be the same as that for entangled photons. Thus the effect seen in the Ghosh-Mandel experiment is essentially the HBT effect. As the interference in the HBT effect is independent of whether the two particles are entangled or not, the Ghosh-Mandel experiment is not a demonstration of non-local quantum correlation or entanglement between photons, as many seem to believe [37, §6.5]. However, in the light of our analysis, the Ghosh-Mandel experiment was historically the first unambiguous demonstration of interference between two photons, which is a completely non-classical effect in itself, and probably would not have been expected by Dirac [3]. A word of caution might be needed here. The HBT effect should not be naively considered a consequence of just a physical overlap of two wave-packets of two photons [39]. It should rather be thought of as interference of two two-photon amplitudes.

\section{Conclusion}

To summarize, we have used wave-packets to study the HBT effect in quantum particles following BoseEinstein and Fermi-Dirac statistics, using a recently introduced label-free analysis of indistinguishable particles. The bunching and anti-bunching has been demonstrated through a simple analysis. We have also analyzed the HBT effect for pairs of particles which are entangled in position and momentum through an EPR like state. These entangled particles also show an HBT effect which is not different from the HBT effect in independent particles, in any noticeable way.

We have also argued that the Ghosh-Mandel experiment is essentially the HBT effect with entangled particles. However, the interference seen in that experiment, is not a consequence of any non-local correlation between the two photons. Exactly the same effect would be observed if the photons were not entangled.

\section{Acknowledgments}

This work is inspired by the lucid exposition of the HBT effect by N. D. Hari Dass. Ushba Rizwan thanks the Centre for Theoretical Physics for providing her the facility of the Centre during the course of this work.

\section{References}

[1] Hanbury Brown R, Twiss RQ. A new type of interferometer for use in radio astronomy. Philosophical Magazine 1954; 45(366): 663-682. doi : 10.1080/ 14786440708520475

[2] Born M, Wolf E. Principles of Optics: Electromagnetic Theory of Propagation, Interference and Diffraction of Light, 7th edition. Cambridge: Cambridge University Press, 2003.

[3] Dirac PAM. The Principles of Quantum Mechanics, 4th edition. Oxford: Oxford University Press, 1967. https://archive.org/details/ DiracPrinciples0fQuantumMechanics

[4] Hanbury Brown R, Twiss RQ. Correlation between photons in two coherent beams of light. Nature 1956; 177(4497): 27-29. doi:10.1038/ $177027 a 0$

[5] Fano U. Quantum theory of interference effects in the mixing of light from phase-independent sources. American Journal of Physics 1961; 29(8): 539-545. doi:10.1119/1.1937827

[6] Mandel L. Quantum effects in one-photon and two-photon interference. Reviews of Modern Physics 1999; 71(2): S274-S282. doi:10.1103/ RevModPhys.71.S274

[7] Kaltenbaek R, Blauensteiner B, Zukowski M, Aspelmeyer M, Zeilinger A. Experimental interference of independent photons. Physical Review Letters 2006; 96(24): 240502. arXiv:quantph/0603048, doi:10.1103/PhysRevLett.96. 240502

[8] Yasuda M, Shimizu F. Observation of two-atom correlation of an ultracold neon atomic beam. Physical Review Letters 1996; 77(15): 3090-3093. doi: 10.1103/PhysRevLett.77.3090 
[9] Schellekens M, Hoppeler R, Perrin A, Gomes JV, Boiron D, Aspect A, Westbrook CI. Hanbury Brown Twiss effect for ultracold quantum gases. Science 2005; 310(5748): 648-651. doi : 10.1126/ science. 1118024

[10] Öttl A, Ritter S, Köhl M, Esslinger T. Correlations and counting statistics of an atom laser. Physical Review Letters 2005; 95(9): 090404. doi: 10.1103/PhysRevLett. 95.090404

[11] Neder I, Ofek N, Chung Y, Heiblum M, Mahalu $\mathrm{D}$, Umansky V. Interference between two indistinguishable electrons from independent sources. Nature 2007; 448: 333-337. doi:10.1038/ nature05955

[12] Li YS, Zeng B, Liu XS, Long GL. Entanglement in a two-identical-particle system. Physical Review $A$ 2001; 64(5): 054302. arXiv:quant-ph/0104101, doi:10.1103/PhysRevA.64.054302

[13] Paškauskas R, You L. Quantum correlations in twoboson wave functions. Physical Review A 2001; 64(4): 042310. arXiv: quant-ph/0106117, doi: 10.1103/PhysRevA.64.042310

[14] Schliemann J, Cirac JI, Kuś M, Lewenstein M, Loss D. Quantum correlations in two-fermion systems. Physical Review A 2001; 64(2): 022303. arXiv: quant-ph/0012094, doi:10.1103/PhysRevA. 64.022303

[15] Eckert K, Schliemann J, Bruß D, Lewenstein M. Quantum correlations in systems of indistinguishable particles. Annals of Physics 2002; 299(1): 88127. arXiv:quant-ph/0203060, doi:10.1006/ aphy .2002.6268

[16] Plastino AR, Manzano D, Dehesa JS. Separability criteria and entanglement measures for pure states of $N$ identical fermions. Europhysics Letters 2009; 86(2): 20005. arXiv: 1002 . 0465, doi : 10.1209/ 0295-5075/86/20005

[17] Ghirardi G, Marinatto L. General criterion for the entanglement of two indistinguishable particles. Physical Review A 2004; 70(1): 012109. arXiv: quant-ph/0401065, doi:10.1103/PhysRevA. 70.012109

[18] Tichy MC, Mintert F, Buchleitner A. Essential entanglement for atomic and molecular physics. Journal of Physics B: Atomic, Molecular and Optical Physics 2011; 44(19): 192001. arXiv: 1012.3940, doi : 10.1088/0953-4075/44/19/192001
[19] Ghirardi G, Marinatto L, Weber T. Entanglement and properties of composite quantum systems: a conceptual and mathematical analysis. Journal of Statistical Physics 2002; 108(1): 49122. arXiv:quant-ph/0109017, doi : 10.1023/ a: 1015439502289

[20] Tichy MC, de Melo F, Kuś M, Mintert F, Buchleitner A. Entanglement of identical particles and the detection process. Fortschritte der Physik 2013; 61(23): 225-237. arXiv:0902 . 1684, doi:10.1002/ prop. 201200079

[21] Buscemi F, Bordone P, Bertoni A. Linear entropy as an entanglement measure in two-fermion systems. Physical Review A 2007; 75(3): 032301. arXiv: quant-ph/0611223, doi:10.1103/PhysRevA. 75.032301

[22] Reusch A, Sperling J, Vogel W. Entanglement witnesses for indistinguishable particles. Physical Review A 2015; 91(4): 042324. arXiv: 1501.02595, doi : 10.1103/PhysRevA.91.042324

[23] Shi Y. Quantum entanglement of identical particles. Physical Review A 2003; 67(2): 024301. arXiv: quant-ph/0205069, doi:10.1103/PhysRevA. 67.024301

[24] Benenti G, Siccardi S, Strini G. Entanglement in helium. European Physical Journal D 2013; 67(4): 83. arXiv: 1204.6667, doi : 10.1140/epjd/e201340080-y

[25] Balachandran AP, Govindarajan TR, de Queiroz AR, Reyes-Lega AF. Entanglement and particle identity: a unifying approach. Physical Review Letters 2013; 110(8): 080503. arXiv: 1303.0688, doi:10.1103/PhysRevLett.110.080503

[26] Benatti F, Floreanini R, Marzolino U. Bipartite entanglement in systems of identical particles: the partial transposition criterion. Annals of Physics 2012; 327(5): 1304-1319. arXiv: 1202 . 2993, doi : 10, $1016 / \mathrm{j}$.aop. 2012.02.002

[27] Sasaki T, Ichikawa T, Tsutsui I. Entanglement of indistinguishable particles. Physical Review A 2011; 83(1): 012113. arXiv: 1009.4147, doi: 10.1103/PhysRevA.83.012113

[28] Benatti F, Floreanini R, Marzolino U. Entanglement robustness and geometry in systems of identical particles. Physical Review A 2012; 85(4): 042329. arXiv:1204.3746, doi:10, 1103/PhysRevA.85.042329 
[29] Benatti F, Floreanini R, Titimbo K. Entanglement of identical particles. Open Systems $\mathcal{E}$ Information Dynamics 2014; 21(1-2): 1440003. arXiv: 1403. 3178, doi:10.1142/s1230161214400034

[30] Styer DF, Balkin MS, Becker KM, Burns MR, Dudley CE, Forth ST, Gaumer JS, Kramer MA, Oertel DC, Park LH, Rinkoski MT, Smith CT, Wotherspoon TD. Nine formulations of quantum mechanics. American Journal of Physics 2002; 70(3): 288297. doi : 10.1119/1.1445404

[31] Lo Franco R, Compagno G. Quantum entanglement of identical particles by standard informationtheoretic notions. Scientific Reports 2016; 6: 20603. arXiv: 1511.03445, doi : 10.1038/srep20603

[32] Lévy-Leblond J-M. Quantum words for a quantum world. In: Epistemological and Experimental Perspectives on Quantum Physics. Greenberger D, Reiter WL, Zeilinger A (editors), Dordrecht: Springer, 1999, pp. 75-87. doi : 10.1007/978-94017-1454-9_5

[33] Einstein A, Podolsky B, Rosen N. Can quantummechanical description of physical reality be considered complete? Physical Review 1935; 47(10): 777-780. doi: 10.1103/PhysRev.47.777

[34] Qureshi T. Understanding Popper's experiment.
American Journal of Physics 2005; 73(6): 541544. arXiv:quant-ph/0405057, doi:10.1119/ 1.1866098

[35] Qureshi T, Shafaq S. Wave-packet analysis of singleslit ghost diffraction. European Physical Journal Plus 2015; 130(8): 173. arXiv: 1505.05559, doi: 10.1140/epjp/i2015-15173-6

[36] Ghosh R, Mandel L. Observation of nonclassical effects in the interference of two photons. Physical Review Letters 1987; 59(17): 1903-1905. doi : 10 . 1103/PhysRevLett.59.1903

[37] Greenstein G, Zajonc AG. The Quantum Challenge: Modern Research on the Foundations of Quantum Mechanics, 2nd edition. Sudbury, Massachusetts: Jones \& Bartlett Publishers, 2005.

[38] Chan KW, Torres JP, Eberly JH. Transverse entanglement migration in Hilbert space. Physical Review A 2007; 75(5): 050101. arXiv:quant-ph/ 0608163, doi : 10.1103/PhysRevA.75.050101

[39] Pittman TB, Strekalov DV, Migdall A, Rubin MH, Sergienko AV, Shih YH. Can two-photon interference be considered the interference of two photons? Physical Review Letters 1996; 77(10): 1917-1920. doi:10.1103/PhysRevLett.77.1917 